\title{
Status of Medicinal and Aromatic Plants in the State of Jammu and Kashmir, India
}

\author{
Sonam Dawa ${ }^{1}$, Padma Gurmet ${ }^{2}$, Tsewang Dolma ${ }^{2}$, Tsering Angdus ${ }^{2}$, \\ Tashi Stobgais ${ }^{2}$ and Thinles Tharpa ${ }^{2}$ \\ ${ }^{1}$ Division of Floriculture and Landscape Architecture, SKUAST-K Srinagar, India \\ ${ }^{2}$ National Research Institute for Sowa-Rigpa-Leh-Ladakh, (Ministry of AYUSH), India \\ *Corresponding author
}

\begin{abstract}
A B S T R A C T
India is home to a great variety of ethno-medicinally important plant species and is ranked sixth among 12 mega-diversity countries of the world (Joshi et al., 2001). Medicinal plants

Keywords

Medicinal plants,

Biodiversity,

Himalayan

Article Info

Accepted:

17 November 2018

Available Online:

10 December 2018 as a group comprises approximately 8000 species and account for around $80 \%$ of all higher flowering plant species of India (Samant et al., 2002). The Indian Himalayan Region (IHR) comprises of five biogeographic provinces i.e. Trans, North West, West, Central and East Himalaya and covers approximately an area of 591 thousand $\mathrm{km}^{2}$ (Rodgers and Panwar, 1998). The Himalayas are recognised as one of the global biodiversity hotspots where ecological, phyto-geographical and evolutionary factors favours high species diversity (Samant et al., 2003). It has an large altitudinal range (300$800 \mathrm{~m}$ amsl) and supports a unique flora and fauna. About 18,440 species of plants $(25.3 \%$ species endemic), 1748 species of medicinal plants (Samant et al., 2003) and 675 species of wild edibles (Samant and Dhar, 2002). Medicinal and aromatic plants (MAPs) are traded as both as raw material and as processed final products. The collection and more recently marketing of (MAPs) has provided an important source of income for communities living in the mountain areas (Rodgers and Panwar, 1998). The sustainably use of such plants in tied closely to future opportunities for high altitudes Himalayan

\section{Introduction}

The prehistoric men and women, as they advanced towards civilization in the lap of the time, depended on plants. With the progress of civilization from the dim past, even before the present century, various charms of the plants played a great role in human life, society and activities. In Egypt, India and China mines of information are available in the old literature, folklores, mythological stories, epic poems, medicinal treaties, thousands of years old

manuscripts, copper plates leaves and similar other records, many of which are kept preserved even to the present day. A vast field of knowledge on this subject still remains terra incognita in the innumerable Tibetan manuscripts and writing on herbal preserved in the archives or the monasteries scattered all over Sikkim, Bhutan and Tibet. The earliest reference to the use of medicinal herbs as a cure for diseases and as charms is found in the manuscripts of "Eber Papyrus" which dates to about $16^{\text {th }}$ century B.C.
\end{abstract}


The mysticism of the medicinal plants is also mentioned in the Atharva and Rig Vedas. Perhaps the oldest repositories of human knowledge were written between 400 and 1600 B.C. by Charaka and Susruta in the treatment of maladies in human being and animals. These are monumental work and outstanding contributions towards our knowledge of science and art of healing. Later during the Buddhist period, considerable progress was made and medicinal plants were cultivated under the direction of highly qualified specialists. Out of 200 items recorded in Indian medicinal literature less than 200 are of mineral and animal origin, the rest are derived from vegetable sources. The vegetable materia medica has been built up in the course of centuries and every region of India has contributed to its development.

The use of different parts of several medicinal plants to cure specific ailment has been in vogue from ancient times. The indigenous system of medicine namely Ayurvedic, Siddha and Unani have been in existence for several centuries. This system of medicines caters to the needs of nearly 70 per cent of our population residing in the villages. Apart from India, these systems of medicine are prevalent in China, Korea, Singapore, Malaysia, West Asia and many other countries. Besides the demands made by the modern pharmaceutical industries has also increased manifold.

India is home to a great variety of ethnomedicinally important plant species, and is ranked sixth among 12 mega-diversity countries of the world. Medicinal plants as a group comprise approximately 8000 species and account for around $50 \%$ of all higher flowering plant species of India. The Himalayas is recognised as one of the global biodiversity hotspots, where ecological, phytogeographical and evolutionary factors favour high species diversity. A bio-geographically unique region, it has the highest degree of species endemism in the Asian region it supports about 18,440 species of plants, of which $25.3 \%$ are endemic to the region, a high proportion of which are of use for medicinal purposes. Medicinal and aromatic plants (MAPs) are traded both as raw materials and as processed final products. Demand for a wide variety of species is increasing as these markets expand and new end-uses are developed. The price trends of most of the Himalayan species of medicinal plants traded in market have been upwards in the last 3 years (Table 1 and 2).

However, about $90 \%$ of medicinal plants used by the industries are collected from the wild. While over 800 species are used in the industry, less than 20 species of plants are under commercial cultivation. The increase in demand increases the threat of depletion, and there are currently seventeen Himalayan medicinal plant species listed in the Indian government Red Data Book of Indian Plants). The collection and, more recently, marketing of these plants has provided an important source of income for communities living in mountain areas. The ability to sustainably use such plants is tied closely to future opportunities for high altitude Himalayan communities.

The Himalayan region is diverse also in traditions of medical practice (Table 4). Apart from the Ayurvedic (Indian) tradition, the Unani (Islamic) and Sowa-rigpa (Tibetan) systems of medicine, as well as diverse folk traditions, are practised in the region. The international market for medicinal and aromatic plants (MAPs) is dominated by China, Japan, five European countries and the USA. Within Europe there are good reasons to focus on the UK as the primary target for new sales of these products from India. The shape of the UK market is here outlined. Current sales into Europe from India are relatively low. 


\section{Production of medicinal herbs}

Generally, the villagers acquire basic knowledge about the use of various herbs from their elders and collect them fresh from their gardens or nearby forests, whenever they need them. However, with deforestation and commercialization of agriculture, many medicinal herbs are not easily available. Furthermore, with the development of allopathic systems, doctors discourage their patients from using herbal medicines. Hence, most of the Ayurvedic medicines produced by the pharmaceutical firms are used as backup remedies, tonics or to counteract the side effects of allopathic medicines, resulting in loss of faith in the system.

Presently, a large number of firms are involved in bulk production of herbal medicines in India. As most of these firms do not have their own source of raw materials required for processing, they are dependent on the natural forests.

These firms have been engaging the local forest dwellers to collect raw material from the forests. The estimated value of herbs extracted for drug production is over US\$300 million. The raw material for most of these products comes from the natural forests for which the industry barely pays the cost of labour. This has resulted in the destruction of a large number of herbal species leading to their extinction. Presently, very few species have been exploited for commercial production in India. Among them, Opium is the most important crop, cultivated for production of morphine over 23000 ha land in U.P., M.P. and Rajasthan.

Senna is another crop cultivated in Tamil Nadu and Andhra Pradesh, covering around 2700 ha, yielding about 5000 tons of leaves and pods. Psyllium (Isabgul) is another important crop grown on 30000 ha in North
Gujarat and adjoining area of Rajasthan, yielding 30,000 ton seeds. Out of this, about $82-85 \%$ of the produce is exported. Periwrinkle (Catharanthus roseus) for extraction of Raubasine, yam bearing Diosgenin (Dioscorea composita) and Sinkona (Cinchona calysaya) are the other important species grown commercially on non-forest lands in India for medicinal properties apart from the large number of herbs and trees having medicinal properties (Table 3 ).

A few more plant species brought under commercial production during recent years are Menthol (Mentha arvensis), Safed musli (Chlorophytum tuberosum), Aloe vera (Aloe barbadensis) and Anola (Emblica officinalis). But in the absence of an assured market and fluctuating price, great uncertainty prevails in the field.

\section{Collection of drugs}

a. Underground parts are storage organs for the plant and accumulate active principles during summer months. Roots and rhizomes of perennials are generally gathered after two to three years of growth. The roots of annuals are generally not collected. This applies to majority of drug plants collected from wild.

b. Tubers, corms and bulbs are collected at the end of flowering or fruiting when all the aerial portions show signs of senescence. All the underground portion are collected, cleaned and dried in shade or ovens at $30^{\circ} \mathrm{C}$ proper care should be taken to dry the materials.

c. Leaves are gathered throughout the growing period. Young leaves are considered to be the highest quality so far as content of active principles is concerned. Herbage or whole herb is collected with flower-bearing stems just before or at the beginning of the flowering stage. Fruits and seeds are collected when mature and should be properly dried. 
d. Bark is collected either in spring, when the trees and shrubs begin to bud or in autumn after they have shed their leaves. The flow of sap is considered to be at maximum at these times and bark readily detaches from the wood. The drugs should be stored properly in dry and cool places taking every precaution to save the material from insect damage and moulds. It should be thoroughly examined before sending to the consumer in industry. The potency of drug plant is dependent upon processing procedures adopted after collection and harvesting from cultivated sources.

\section{Conservation of medicinal and aromatic plants}

Medicinal plant reserves will go a long way to protect the existing genetic diversity and help in rational exploitation of medicinal plant resources in the Himalayas for overall economic development. Priority should be given to species that are endangered throughout the range. Families and genera that are monotypic should receive top priority over polytypic ones. Other things equal, an endangered species should be given priority over the vulnerable one, a vulnerable over a rare one and a rare species over one that even it is declining is considered insufficiently threatened to qualify for one or 3 categories. Creation and development of medicinal plants reserve is a main component of management strategy. A medicinal plant reserve, no matter where it is located in the world must, to some extent, address itself to three basic concepts. In particular it must have one or several protected zones to ensure its "conservation role" which must always be present. It must participate in national and international network and thus have a "logistic role" supporting a certain amount of research work taking part in exchange of information. It must go as far as possible through problem oriented research, demonstration, education and local participation in the "development role". The relative importance of these roles will vary from one medicinal plant reserve to the other, but it is their combined presence which is characteristic of the whole scheme. An area which combines conservation with research and education or sustainable development can only become a true Medicinal Plant Reserve (MPR).

\section{Sustainable management of medicinal and aromatic plant resources}

Sustainable management means using/managing of a species, a group of species or a population or an ecosystem at rate within its capacity for renewal and in a manner compatible with conservation of the diversity and long term viability of the resource and its supporting ecosystem.

\section{Development benefits}

i) Assuring the long term supply of valuable resources

ii) Enabling resources depleted by overuse to recover

iii) Regulate international trade by assuring supply of quality materials

iv) Ensure that excess to a resources does not exceed the resources capacity to sustain exploitation.

v) Overall development of resources available in the ecosystem.

\section{Conservation benefits}

Conservation not only the species concerned but also its supporting ecosystems and the species associated with these ecosystem.

\section{Social and economic benefits}

The sustainable use can provide incentive to conserve wild species and their supporting ecosystems, provided the people most likely to have an impact on the species and ecosystem concerned receives an adequate share of the benefits 
Table.1 Demand of medicinal herbs and their availability

\begin{tabular}{|c|c|c|c|}
\hline $\begin{array}{l}\text { High demand/ } \\
\text { High supply, } \\
\text { Equal demand supply } \\
\text { relation }\end{array}$ & $\begin{array}{l}\text { High demand/ } \\
\text { Low supply; } \\
\text { demand greater than } \\
\text { supply }\end{array}$ & $\begin{array}{l}\text { Low demand/ } \\
\text { High supply, } \\
\text { Demand lower than } \\
\text { supply }\end{array}$ & $\begin{array}{l}\text { Low demand/ } \\
\text { Low supply, } \\
\text { Equal Demand supply } \\
\text { relation }\end{array}$ \\
\hline $\begin{array}{l}\text { Usnea longissima/ } \\
\text { Usnea sikkimensis, } \\
\text { Allium carolinianum, } \\
\text { Betula utilis, } \\
\text { Cedrus deodara, } \\
\text { Valeriana jatamansi, }\end{array}$ & $\begin{array}{l}\text { Aconitum } \\
\text { Heterophyllum, } \\
\text { Taxus baccata } \\
\text { subsp. wallichiana, } \\
\text { Podophyllum } \\
\text { hexandrum, } \\
\text { Swertia chirayita, } \\
\text { Nardostachys } \\
\text { Grandiflora, } \\
\text { Saussurea costus, } \\
\text { Pleurospermum } \\
\text { angelicoides, } \\
\text { Cordyceps sinensis, } \\
\text { Picrorhiza kurrooa, } \\
\text { Angelica glauca, } \\
\text { Dactylorhiza } \\
\text { hatagirea, }\end{array}$ & $\begin{array}{l}\text { Hippophae } \\
\text { Rhamnoides, } \\
\text { Heracleum } \\
\text { candicans, } \\
\text { Rheum austral, } \\
\text { Pinus roxburghii, } \\
\text { Selenium vaginatum, } \\
\text { Corydalis govaniana, }\end{array}$ & $\begin{array}{l}\text { Berberis aristata, } \\
\text { Urtica dioica, }\end{array}$ \\
\hline
\end{tabular}

Source: Mills et al., (2008)

Table.2 Availability of medicinal plants in different bio-geographical zones of India

\begin{tabular}{|c|c|c|}
\hline Bio-geographical zones & $\begin{array}{l}\text { No. of } \\
\text { known } \\
\text { medicinal plants }\end{array}$ & Occurrence of some important medicinal plants \\
\hline 1. Trans Himalayan zone & 700 & $\begin{array}{l}\text { Ephedra gerardiana, Hippophae rhamnoides, Arnebia } \\
\text { euchroma }\end{array}$ \\
\hline $\begin{array}{l}\text { 2. Himalayan zone } \\
\text { (i) North West Himalaya } \\
\text { (ii) Western Himalaya }\end{array}$ & 1,700 & $\begin{array}{l}\text { Aconitum spp., Berberis spp., Ferula jaeschkeana, } \\
\text { Saussurea costus, Dactylorhiza hatagirea, Picrorhiza } \\
\text { kurroa, Podophyllum hexandrum, Rheum australe, } \\
\text { Swertia chirayita, Taxus wallichiana, Gentiana kurroo, } \\
\text { Inula racemosa. }\end{array}$ \\
\hline $\begin{array}{l}\text { (iii) Central Himalaya } \\
\text { (iv) Eastern Himalaya }\end{array}$ & 1,200 & $\begin{array}{l}\text { Nardostachys grandiflora, Taxus wallichiana, Coptis } \\
\text { teeta, Panax pseudo-ginseng, Swertia chirayita, Rheum } \\
\text { australe,Picrorhiza kurroa, Podophyllum hexandrum, } \\
\text { Gaultheria fragantissima, Entada pursaetha. }\end{array}$ \\
\hline $\begin{array}{l}\text { 3. Desert zones } \\
\text { Kutch and Thar }\end{array}$ & 500 & $\begin{array}{l}\text { Convolvulus microphyllus, Tecomella undulata, } \\
\text { Citrullus colocynthis, Cressa cretica. }\end{array}$ \\
\hline 4. Semi-arid zone & 1,000 & Commiphora wightii, Alhagi pseudalhagi, Salvadora \\
\hline 5. Western Ghats & 2,000 & Myristica malabarica, Cosciniu fenestratum,Garcinia \\
\hline
\end{tabular}




\begin{tabular}{|c|c|c|}
\hline $\begin{array}{l}\text { (i) Western Ghats } \\
\text { mountains } \\
\text { (ii) Malabar coasts }\end{array}$ & & indica, Vateria indica, Utleria salicifolia, \\
\hline $\begin{array}{l}\text { 6. Deccan Peninsula } \\
\text { (i) Deccan Plateau South } \\
\text { (ii) Central Plateau } \\
\text { (iii) Eastern Plateau } \\
\text { (iv) Chota Nagpur } \\
\text { (v) Central Highlands }\end{array}$ & 3,000 & $\begin{array}{l}\text { Pterocarpus santalinus, Mesua ferrea, Decalepis } \\
\text { hamiltonii, Aristolochia spp., Terminalia paliida }\end{array}$ \\
\hline $\begin{array}{l}\text { 7. Gangetic Plains } \\
\text { (i) Upper Gangetic } \\
\text { Plains } \\
\text { (ii) Lower Gangetic } \\
\text { Plains }\end{array}$ & 1,000 & $\begin{array}{l}\text { Holarrhena pubscens, Mallotus phillipinensis, Pluchea } \\
\text { lanceolata, Peganum harmala, Chlorophytum spp., } \\
\text { Rauvolfia serpentine, Saraca asoca }\end{array}$ \\
\hline $\begin{array}{l}\text { 8. North East India } \\
\text { (i) Brahmaputra valley } \\
\text { (ii) Assam hills }\end{array}$ & 2,000 & $\begin{array}{l}\text { Aquilaria malaccensis, Smilax glabra, Abroma augusta, } \\
\text { Hydnocarpus kurzii }\end{array}$ \\
\hline $\begin{array}{l}\text { 9. Islands } \\
\text { (i) Andaman islands } \\
\text { (ii) Nicobar islands } \\
\text { (iii) Lakshdweep islands }\end{array}$ & 1,000 & $\begin{array}{l}\text { Calophyllum inophyllum, Adenanthera pavonina, } \\
\text { Barringtonia asiatica, Aisandra butyracea }\end{array}$ \\
\hline
\end{tabular}

Source: Tiwari, 2008

Table.3 Leading countries of import and export of medicinal and aromatic plants from 20142015

\begin{tabular}{|l|l|l|l|l|l|}
\hline $\begin{array}{l}\text { Country of } \\
\text { import }\end{array}$ & $\begin{array}{l}\text { Volume } \\
\text { [tonnes }]\end{array}$ & $\begin{array}{l}\text { Value } \\
{[1000 \text { US\$ } \$}\end{array}$ & $\begin{array}{l}\text { Country of } \\
\text { export }\end{array}$ & $\begin{array}{l}\text { Volume } \\
\text { [tonnes] }\end{array}$ & $\begin{array}{l}\text { Value } \\
{[1000 \text { US\$] }}\end{array}$ \\
\hline Hong Kong & 73650 & 314000 & China & 139780 & 298680 \\
\hline Japan & 56750 & 146650 & India & 36750 & 57400 \\
\hline USA & 56000 & 133350 & Germany & 15050 & 72400 \\
\hline Germany & 45850 & 113900 & USA & 11950 & 114450 \\
\hline Rep.Korea & 31400 & 52550 & Chile & 11850 & 29100 \\
\hline France & 20800 & 50400 & Egypt & 11350 & 13700 \\
\hline China & 12400 & 41750 & Singapore & 11250 & 59850 \\
\hline Italy & 111450 & 42250 & Mexico & 10600 & 10050 \\
\hline Pakistan & 11350 & 11850 & Bulgaria & 10150 & 14850 \\
\hline Spain & 8600 & 27450 & Pakistan & 8100 & 5300 \\
\hline UK & 7600 & 25550 & Albania & 7350 & 14050 \\
\hline Singapore & 6550 & 55500 & Morocco & 7250 & 13200 \\
\hline Total & $\mathbf{3 4 2 5 5 0}$ & $\mathbf{1 0 1 5 2 0 0}$ & Total & $\mathbf{2 8 1 5 5 0}$ & $\mathbf{6 4 3 2 0 0}$ \\
\hline
\end{tabular}

Source: Lange, 2002 
Table.4 Five biogeographic provinces of Indian Himalayan Region (IHR)

\begin{tabular}{|c|c|c|c|}
\hline Jammu region & Kashmir region & Leh region & Kargil region \\
\hline $\begin{array}{l}\text { Cymbogon } \\
\text { flexuosus, } \\
\text { C. winterianus, } \\
\text { C. maritinii, } \\
\text { Vetiveria } \\
\text { zizanoides, } \\
\text { Pelargonia } \\
\text { graveolens, } \\
\text { Eucalyptus } \\
\text { citriodes, } \\
\text { Argemone } \\
\text { graveolens, } \\
\text { Allium stoliczka, } \\
\text { A. victoralis, } \\
\text { Withania } \\
\text { somnifera, } \\
\text { Matricaria } \\
\text { chamomilla, } \\
\text { Bacopa } \\
\text { monnieri, } \\
\text { Andrographis } \\
\text { paniculate }\end{array}$ & 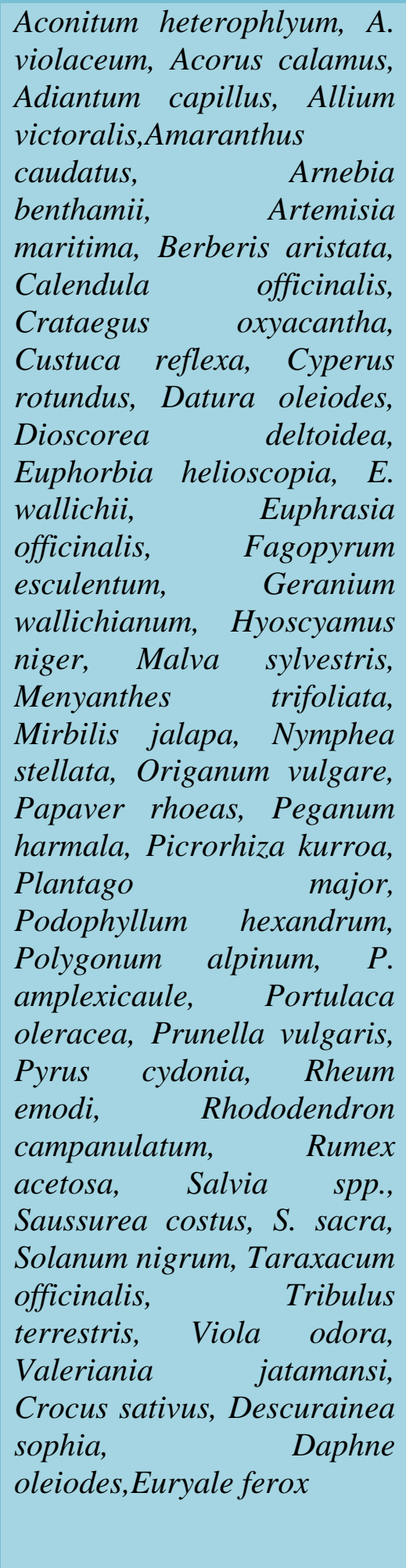 & 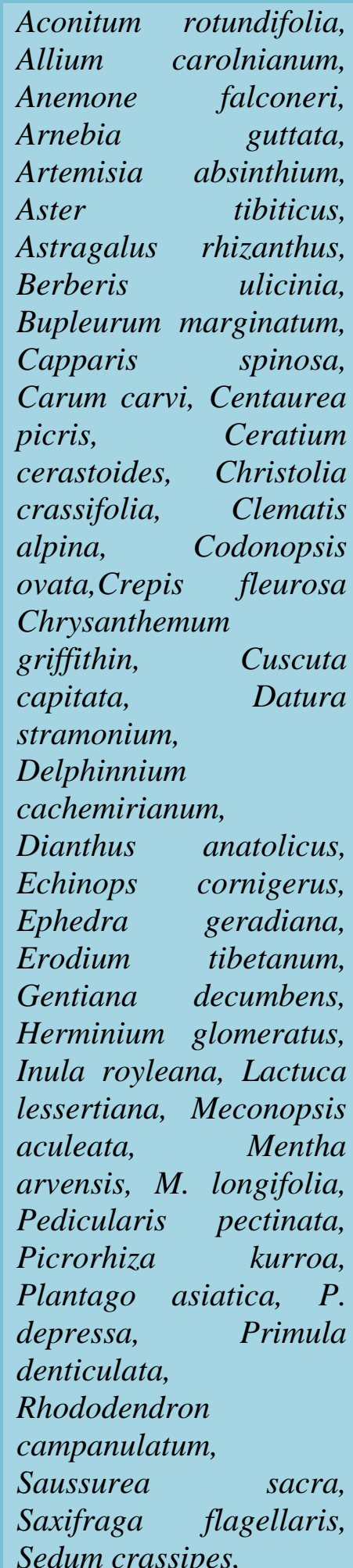 & $\begin{array}{l}\text { Achillea millefolia, } \\
\text { Aconitum } \\
\text { heterophyllum } \\
\text { Arnebia enchroma, } \\
\text { Artemisia } \\
\text { dracunculas, } \\
\text { A.japonica, } \\
\text { A.laciniata, Berginia } \\
\text { strachyi, Carum carvi, } \\
\text { Chenopodium botrys, } \\
\text { Cicer microphyllum, } \\
\text { Codonopsis } \\
\text { clematidea, } \\
\text { Coriandum sativum, } \\
\text { Geraniumwallichiana, } \\
\text { Hyoscyamus niger, } \\
\text { Inula racemosa, } \\
\text { Juniperus communis, } \\
\text { J. macropoda, Malva } \\
\text { parvifolia,Podophyllu } \\
\text { m hexandrum } \\
\text { Polygonum aviculare, } \\
\text { Prangos pabularia, } \\
\text { Rosa webbiana, } \\
\text { Senecio } \\
\text { jacquemontianus, } \\
\text { Sisybrium } \\
\text { brassiciforme, Stachys } \\
\text { tibetica, Thymus } \\
\text { serpyllum, Vicia sativa }\end{array}$ \\
\hline
\end{tabular}


Table.5 Chemical composition of some important medicinal plants

\begin{tabular}{|l|l|}
\hline Plant name & Chemical constituents \\
\hline Acorus calamus & Calamen, calamenol \\
\hline Taxus baccata & Taxol \\
\hline Artemisia absinthium & Sesquiterpene. artabsin, arabsin \\
\hline Berberis aristata & Berberine \\
\hline Cannabis sativa & Cannabinol, cannabinin \\
\hline Carum carvi & Carvone \\
\hline Ephedra geradiana & Ephedrine \\
\hline Hyoscyamus niger & Hyoscyamine \\
\hline Phytolaca acinosa & Phytolaca toxin \\
\hline Picrorhiza kurroa & Picrorhizin \\
\hline Podophyllum hexandrum & Podophyllin, podophyllotoxin \\
\hline Digitalis purpurea & Digitoxin \\
\hline Colchicum luteum & Colchicine \\
\hline
\end{tabular}

Source: Kaul, 2001

Table.6 Plants used medicinally worldwide

\begin{tabular}{|l|l|l|l|}
\hline Country & Plant species & $\begin{array}{l}\text { Medicinal } \\
\text { species }\end{array}$ & plant \\
\hline China & 26092 & 4941 & 18.9 \\
\hline India & 15022 & 3000 & 20.0 \\
\hline Indonesia & 22500 & 1000 & 4.4 \\
\hline Malaysia & 15500 & 1200 & 7.7 \\
\hline Nepal & 6973 & 700 & 10.0 \\
\hline Pakistan & 4950 & 300 & 6.1 \\
\hline Philippines & 8931 & 850 & 9.5 \\
\hline USA & 21641 & 2564 & 11.8 \\
\hline Average & 13366 & 1700 & 12.5 \\
\hline World & $\mathbf{4 2 2 0 0 0}$ & $\mathbf{5 2 8 8 5}$ & 100 \\
\hline Sord & & & \\
\hline
\end{tabular}

Sources: Govaerts (2001); Padua et al., (1999)

Table.7 Some common medicinal plants and main therapeutic uses of their different plant parts

\begin{tabular}{|l|l|l|l|}
\hline Plant Family & Plants & $\begin{array}{l}\text { Plant parts } \\
\text { used }\end{array}$ & $\begin{array}{l}\text { Main therapeutic } \\
\text { use/ailments treated }\end{array}$ \\
\hline Anacardiceae & Pistachia lentiscus L & Fruits & Rheumatism \\
\hline Araliaceae & Hedera helix L. & Leaf & $\begin{array}{l}\text { Rheumatism, Anti- } \\
\text { inflammatory, burns }\end{array}$ \\
\hline
\end{tabular}




\begin{tabular}{|c|c|c|c|}
\hline Boraginaceae & Boraga officinalis L. & Leaf & $\begin{array}{l}\text { Stomach pain, intestinal } \\
\text { regularization, } \\
\text { diuretic, }\end{array}$ \\
\hline Cactaceae & Opuntia ficus-indica L. & Cladophyll & Skin emollient \\
\hline Caprifoliaceae & Lonicera implexa & Leaf & Diuretic \\
\hline \multirow[t]{4}{*}{ Compositae } & Anthemis arvensis L. & $\begin{array}{l}\text { Whole } \\
\text { plant }\end{array}$ & $\begin{array}{l}\text { Anti-inflammatory, emetic, } \\
\text { sedative }\end{array}$ \\
\hline & Artemisia arborescens L. & Flower & $\begin{array}{l}\text { Digestive, } \\
\text { expectorant }\end{array}$ \\
\hline & Calendula arvensis $\mathrm{L}$. & Flower leaf & $\begin{array}{l}\text { Anti-spasmodic, burns, } \\
\text { diuretic, disinfectant }\end{array}$ \\
\hline & Helychrysum microphyllum & Leaf flower & Expectorant \\
\hline Convolvulaceae & Convolvulus althaeiodes L. & $\begin{array}{l}\text { Whole } \\
\text { plant }\end{array}$ & Fat digestion \\
\hline \multirow[t]{13}{*}{ Labiatae } & Lavandula stoechas & Leaf & $\begin{array}{l}\text { Asthma, headache, } \\
\text { palpitation }\end{array}$ \\
\hline & Melissa officinalis L. & Leaf & $\begin{array}{l}\text { Digestive, lenitive, bad } \\
\text { breath }\end{array}$ \\
\hline & Mentha rotundifolia $\mathrm{L}$. & $\begin{array}{l}\text { Aerial } \\
\text { parts }\end{array}$ & Digestive, lenitive \\
\hline & Mentha spp. & Leaf & Anti-inflammatory, sedative, \\
\hline & Ocimum basilicum $\mathrm{L}$ & Leaf & Anti-inflammatory \\
\hline & Plants & $\begin{array}{l}\text { Plant parts } \\
\text { used }\end{array}$ & $\begin{array}{l}\text { Main therapeutic } \\
\text { use/ailments treated }\end{array}$ \\
\hline & Allium cepa $L$. & Bulb & $\begin{array}{l}\text { Renal calculus, } \\
\text { antispasmodic }\end{array}$ \\
\hline & Allium sativum $L$. & $\begin{array}{l}\text { Bulb, } \\
\text { leaf }\end{array}$ & $\begin{array}{l}\text { Hypotensive, diuretic, } \\
\text { stomach } \\
\text { pain, antibacterial, anti } \\
\text { inflammatory }\end{array}$ \\
\hline & Asparagus acutifolius L. & shoot & Diuretic, gout \\
\hline & Ruscus aculeatus $L$. & $\begin{array}{l}\text { Rhizome, } \\
\text { leaf }\end{array}$ & $\begin{array}{l}\text { Gout, hemorrhoids, anti- } \\
\text { verrucose }\end{array}$ \\
\hline & Linum usitatissimum L. & Seed & $\begin{array}{l}\text { Gout, anti-inflammatory, } \\
\text { laxative, gentle laxative, } \\
\text { erythema }\end{array}$ \\
\hline & Malva sylvestris $L$ & $\begin{array}{l}\text { Whole } \\
\text { plant, } \\
\text { leaf }\end{array}$ & $\begin{array}{l}\text { Expectorant, anti- } \\
\text { inflammatory, } \\
\text { laxative, emollient, eye } \\
\text { inflammation, pressure } \\
\text { regulate }\end{array}$ \\
\hline & Ficus carica $L$. & Leaf & Antiverrucose, \\
\hline
\end{tabular}




\begin{tabular}{|c|c|c|}
\hline & & $\begin{array}{l}\text { sedative, } \\
\text { anti-inflammatory }\end{array}$ \\
\hline $\begin{array}{l}\text { Eucalyptus globules } \\
\text { Labill. }\end{array}$ & Leaf & Anti-catarrhal, expectorant \\
\hline Papaver rhoeas $L$. & $\begin{array}{l}\text { Leaf, } \\
\text { flower }\end{array}$ & Sedative, analgesic, gout \\
\hline $\begin{array}{l}\text { Adiantus capillus veneris } \\
\text { L. }\end{array}$ & Leaf & Diuretic, sedative, emollient \\
\hline Polypodium vulgare $L$. & Rhizome & Cough sedative \\
\hline Crataegus monogyna & Flower & Diuretic, cardiac sedative \\
\hline Rosa canina $L$ & Fruit & $\begin{array}{l}\text { Diarrhoea, } \\
\text { reconstituent }\end{array}$ \\
\hline Rubus fruticosus $L$. & Leaf & Diarrhoea, dysentery \\
\hline Citrus limon $L$ & Fruit & $\begin{array}{l}\text { Disinfectant, hypotensive, } \\
\text { Headache }\end{array}$ \\
\hline Apium graveolens $L$ & Seed & Anti-inflammatory \\
\hline Daucus carota $L$. & Seed & Intestinal analgesic \\
\hline Foeniculum vulgare & $\begin{array}{l}\text { Fruit, } \\
\text { seed, leaf }\end{array}$ & $\begin{array}{l}\text { Digestive, galactogen, } \\
\text { carminative, sedates nausea }\end{array}$ \\
\hline Verbena officinalis $L$. & $\begin{array}{l}\text { Whole } \\
\text { plant }\end{array}$ & $\begin{array}{l}\text { Inappetence, } \\
\text { diseases, } \\
\text { sedative, } \\
\text { cholagogue }\end{array}$ \\
\hline Vitis vinifera $L$. & Fruit & Cough sedative \\
\hline
\end{tabular}

Source: Tiwari, 2008

Table.8 Medicinal plants species threatened worldwide

\begin{tabular}{|l|l|}
\hline Number of flowering plant species worldwide & $\mathbf{4 2 2 0 0 0}$ \\
\hline $12.5 \%$ of them are used medicinally & 52000 \\
\hline $8 \%$ are threatened & 4160 \\
\hline
\end{tabular}

Source: Walter, 1998., Govaert, 2001

Importance of medicinal and aromatic plants

Long before the development of modern medicines, India in ancient times, was entirely dependent on herbal medicines for health care. Many scripts carry elaborate prescriptions of herbal medicines for treating very complex diseases. India was the leader in health care through Ayurveda. Apart from such systematic therapies, specific herbal therapies developed by individuals were used for treating the sick and these secret therapies were passed on from the older generation to the younger generation, without disclosing them to the outsiders.

Even today, herbal medicines play a significant role in India. A similar practice 
was also prevalent in China and many other countries in Asia and Africa. With the advancement of science, allopathic medicine gained prominence over herbal medicines. With larger commercial interest, the western countries promoted modern medicine, in spite of its high cost and side effects. However, realizing the drawbacks of allopathic medicine, the world is turning back to herbal medicines. Presently, about $80 \%$ of the world population is still dependent on medicinal plants for health care and $20 \%$ of the drugs in pharmaceutical firms are of plant origin, either extracted from the plants or synthetic derivatives of these plant species. In China, over 5000 plant species are used in drugs and traditional medicines, while over 2000 species are known for their traditional medicinal value in India. In addition to medicinal properties, some of these species are also used as tonics. Ginsing, one of the most popular species used as a tonic, is widely grown in China and Korea. In 1991, the value of Ginsing sold in USA and Asian region was over US\$ 215 million and China had a lion share of $40 \%$ (Sial, 1995). The estimated annual market value of the plant based drug is US\$ 43,000 million and it is expected to rise to US\$ 3 trillion over the next two decades.

In India, for over 500 million people, traditional herbal medicines are the only alternative source, due to easy accessibility and lower price. This sector also provides employment to over one million traditional healers and Vaidyas in the country (Shankar, 1997). Thus, sustainable development of medicinal herbs provides an excellent opportunity to take advantage of the expanding market, while ensuring a steady supply to local communities.

\section{Strategy for development of herbal medicine industry}

Presently, over 100 million ha of wastelands are not only lying idle, depriving the poor of opportunities for income generation but also posing a serious threat to the ecosystem and environment. Such lands can be used for growing medicinal herbs, which are in good demand. Commercial cultivation of medicinal herbs can be promoted by establishing a linkage with bulk consumers. For successful promotion of herbal medicine industry in the country, the following steps are suggested.

Identification of plant species having medicinal properties and establishment of data banks and herbariums.

Conservation of medicinal herbs in their natural habitats -- in-situ and in protected areas (ex-situ)

Selection of superior germplasm based on laboratory analysis and field studies, for domestication and commercial production.

Standardization of propagation and cultivation practices for producing superior quality herbal materials, which can be adopted by farmers.

Validation of the uses of various medicinal herbs in curing different diseases, standardization of the protocols and documentation.

Strengthening of research to find suitable herbal medicines to cure various diseases, particularly newly emerging diseases.

Patenting of the traditional practices and germplasm. Facilitation counters can be established to assist the traditional healers to patent their therapy.

Introduction of herbal medicine as an integral subject in medical colleges dealing with different systems.

Popularizing the use of various herbal medicines through specific prescriptions, social marketing and mass awareness.

Supporting traditional healers and Ayurvedic practitioners through sharing of knowledge, networking, supply of superior quality germplasm and linkage with farmers for supply of raw materials. 
Establishing a formal linkage between the cultivators, bulk consumers and pharmaceutical industries at national and international levels.

Modifications in the Government policies to support research and development on herbal medicines and to curb over-exploitation of medicinal herbs in natural forests.

\section{General classifications of medicinal plants on the basis of their uses are}

Used by traditional ones and herbal patricians.

Used in formulation of different Ayurvedic products.

Used for making herbal products.

Used in synthetic medicinal formulation.

Used for extraction of their active moiety.

Used in other than pharmaceutical industries.

With development of pharmaceutical industries much more attendance has been created on plant products. They have attendance to isolated active constituents from different plant parts and use them directly as drug or design them as pharmacologically active compounds with or without addition of synthetic ones.

In India traditional communities like tribal and rural populations are frequently using the crude extracts of local plants for medicinal and other purposes. Crude extracts and medicines manufactured on the principles of natural compounds even by pharmaceuticals companies, may lead to large-scale exposure of humans to natural products (Table 6).

\section{Challenges in medicinal plants sector}

The continuous increase in human population is one of the causes for concern in meeting the daily requirements of food and medicine as the economy and livelihoods of human societies living in developing countries primarily depend on forest products. This phenomenon is leading to continuous erosion of forest and the forest products, thus making challenge to meet the requirements as well as to conserve useful bio-resources.

More and more species are being gradually added in the Materia Medica; however, the standards of their purity and correct identification do not keep pace with the process of expansion. The market prices for medicinal plants and derived materials provide only a limited insight into the workings of the market, and not on the precise information of profits, supply and demand. We have identified the following major features and challenges on the basis of examining the existing knowledge on the medicinal plants sector.

\section{Rising demand}

The World Health Organization (WHO) has estimated the present demand for medicinal plants is approximately US $\$ 14$ billion per year. The demand for medicinal plant-based raw materials is growing at the rate of 15 to $25 \%$ annually, and according to an estimate of WHO, the demand for medicinal plants is likely to increase more than US $\$ 5$ trillion in 2050. In India, the medicinal plant related trade is estimated to be approximately US \$1 billion per year. For example, the large quantity of Himalayan yew (Taxus baccata) has been gathered from the wild since its extract, taxol, was established as a use in the treatment of ovarian cancer. Aconitum heterophyllum, Nardostachys grandiflora, Dactylorhiza hatagirea, Polygonatum verticillatum, Gloriosa superba, Arnebia benthamii and Megacarpoea polyandra are other examples of north Indian medicinal plant species which have been overexploited for therapeutic uses and have subsequently been placed today in rare and endangered 
categories (Table 7). Many medicinal plant species are used in curing more than one disease, and as a result, these species are under pressure due to over collection from wild. For example, Hemidesmus indicus is used to cure 34 types of diseases; Aegle marmelos, Phyllanthus emblica, and Gloriosa superb. Over-exploitation and continuous depletion of medicinal plants have not only affected their supply and loss of genetic diversity, but have seriously affected the livelihoods of indigenous people living in the forest margins.

More than $95 \%$ of the 400 plant species used in preparing medicine by various industries are harvested from wild populations in India. Harvesting medicinal plants for commercial use, coupled with the destructive harvest of underground parts of slow reproducing, slow growing and habitat-specific species, are the crucial factors in meeting the goal of sustainability.

Harvesting shoots and leaves of medicinal plants may decline their photosynthetic capacity, and as well as the potential for survival and effective propagation. Medicinal plants tolerance to harvest varies with climatic conditions as the temperate herbs become highly vulnerable to harvest of individuals. Furthermore, rising demand with shrinking habitats may lead to the local extinction of many medicinal plant species.

\section{Increasing rarity}

The continuous exploitation of several medicinal plant species from the wild and substantial loss of their habitats during past 15 years have resulted in population decline of many high value medicinal plant species over the years. The primary threats to medicinal plants are those that affect any kind of biodiversity used by humans. The weakening of customary laws, which have regulated the use of natural resources, are among the causes of threatening the medicinal plant species. These customary laws have often proved to be easily diluted by modern socio economic forces.

There are many other potential causes of rarity in medicinal plant species, such as habitat specificity, narrow range of distribution, land use disturbances, introduction of non natives, habitat alteration, climatic changes, heavy livestock grazing, explosion of human population, fragmentation and degradation of population, population bottleneck, and genetic drift. Additionally, natural enemies (i.e., pathogens, herbivores, and seed predators) could substantially limit the abundance of rare medicinal plant species in any given area. In addition to the consumption of medicinal plants by animals, there are physical ailments in humans, which are cured by different species of the same genera. For example, the malarial fever is treated by many species of Swertia (e.g. Swertia chiraiyta, $S$. angustifolia, and $S$. cordata). Similarly, different species of Berberis (e.g. Berberis aristata, B. asiatica, B. lycium, B. chitria and B. jaeschkeana) are used as a source of berberidine to cure certain eye diseases.

Furthermore, different species of the same genera contain different proportions of chemical quantity, and there is a preference over their demand; however, the degree of threat for their exploitation is relatively lower than those species, which do not have alternatives (Table 5). An estimated 4,000 to 10,000 species of medicinal plants face potential local, national, regional or global extinction, with subsequent serious consequences for livelihoods, economies and health care systems. Although, a few studies exist on the rare and endangered medicinal plant species of the northern India, none of these studies have complete data set for even 
a single species. In 2003, 71 rare and endangered medicinal plant species have been assessed for the northwest Himalaya during the Conservation Assessment and Management Plan workshop, according to the guidelines of the World Conservation Union. In northern India, Aconitum is the rarest genus with 5 species, followed by Rheum with 4 rare species. Out of the 71 rare medicinal plants, $92 \%$ are in active trade; $74 \%$ are traded nationally and $35 \%$ are traded internationally.

The meager availability of data on the population and quantum of rare species in nature, however, has restricted their categorization to a few species on the basis of herbarium collection and by consultation by a few experts. The present assessments are also questioned for their validity on the assignment of threat categories to the species, including the number of taxa in danger for specific area. The problems in assessing the species is increased in the mountainous region, especially high altitude areas because of tough and inaccessibility of the terrain, inhospitable climatic conditions, and short life cycle of plants.

Most of the available data have been collected from the easily accessible areas in these mountains. Indigenous communities and commercial herb gatherers also raid these same areas for collection of medicinal plants. Therefore, the estimated population density of categorized rare medicinal plants is not precise because it differs the areas that never and hardly undergone any collection of such rare medicinal plant species.

\section{Cultivation of medicinal plants}

Information on the propagation of medicinal plants is available for less than $10 \%$ and agrotechnology is available only for $1 \%$ of the total known plants globally. This trend shows that developing agro-technology should be one of the thrust areas for research. Furthermore, in order to meet the escalating demand of medicinal plants, farming of these plant species is imperative. Apart from meeting the present demand, farming may conserve the wild genetic diversity of medicinal plants. Farming permits the production of uniform material, from which standardized products can be consistently obtained. Cultivation also permits better species identification, improved quality control, and increased prospects for genetic improvements. Selection of planting material for large-scale farming is also an important task.

The planting material therefore should be of good quality, rich in active ingredients, pestand disease-resistant and environmental tolerant. For the large scale farming, one has to find out whether monoculture is the right way to cultivate all medicinal plants or one has to promote polyculture model for better production of medicinal plants. Studies conducted on the agro-forestry of medicinal plants elsewhere suggest that since many medicinal plant species prefer to grow under forest cover, agroforestry offers a convenient strategy for their cultivation as well as conservation through:

1) Integrating shade tolerant medicinal plants as lower strata species in multistrata system 2) Cultivating short cycle medicinal plants as intercrops in existing stands of tree crops

3) Growing medicinal tree as shade providers and boundary markers, and

4) Inter-planting medicinal plants with food crops.

Out of 10 selected rare and endangered medicinal plant species, Rheum emodi was calculated as a most beneficial cash crop of the medicinal plant in terms of net income generation in northern India. At present, however, the farming of most of the 
medicinal plant species is being operated on a small scale and is restricted to a few hectares of land in various states of northern India. There is an uncertainty of obtaining the necessary permits from government agencies for cultivation of medicinal plants. Additionally, many farmers are unaware about the agency responsible for issuing permits. If the farmers are not granted permits needed to cultivate, they are forced to sell their products on the illegal market, which exposes them to action by government agencies and the exploitation by middlemen.

\section{Overharvesting}

In addition to the loss of access to traditional remedies by indigenous peoples, over collection of species a significant threat to some commercially valuable wild species and to their habitats as well. This threat has been known for decades. According to the World Conservation Union (IUCN), about 15,000 medicinal plant species may be threatened with extinction worldwide from overharvesting. More than 30 years ago, the World Wildlife Fund and the IUCN formed the Wildlife Trade Monitoring Network (TRAFFIC) to monitor and control global trade in wild species, including those used for medicinal plants (Table 8).

\section{Bio-prospecting and bio-piracy}

The former remote green forests have now become part of a dynamic, profit-seeking economy and demanding pluralistic politics worldwide. Reducing the pressure on various forest products, especially on the medicinal plants, is therefore a tough challenge both for policy makers and for economists. Medicinal plants are the local heritage with global importance. The Convention on Biological Diversity at Rio had agreed on a framework that would help the biodiversity to be utilized in a prudent and controlled way. Bio- prospecting, at present, occurs in an environment of suspicions and growing tensions between the biopiracy and rights of sharing benefits between the developing and developed countries.

\section{Strengthening legalized market system}

Besides government agencies, there are numbers of stakeholders ranging from herb gatherers, local middlemen, urban traders, wholesalers, manufacturers, exporters and herbal healers in the medicinal plants trade sector. The marketing system in medicinal plants sector is largely unregulated and inequitable. The medicinal plant collectors are generally the marginal farmers and laborers. They get cash income to meet their basic requirements for food, health and children education by selling medicinal plants. They are often unaware about the real market prices of many medicinal plant species. Generally, in medicinal plants sector, there is a top down approach and even the many stakeholders at the bottom are not aware of the rising demand of their product and the availability of its market. In some villages of Chamoli district of Uttaranchal, the farmers had cultivated Kuth (Saussurea costus) and Dolu (Rheum emodi) but they were unable to sell them due to lack of knowledge on the marketing system. Conversely, many medicinal plant species are traded through illegal channels. The other constraints in the medicinal plants sector are:

1) Slow rate of production of many medicinal plants,

2) Long gestation period

3) Shortage of suitable cultivation technology

4) Production of small quantity

5) Unscientific harvesting

6) Paucity of research on the high yielding varieties

7) Inefficient processing techniques

8) Fluctuation in demand and supply 
9) Poor quality control procedures

10) Scarcity of good manufacturers

11) Poor marketing infrastructure

12) Poor coordination among different stakeholders.

\section{Economic potentialities of some medicinal plants of Ladakh region}

Assumption of the economic potentialities of Suru valley, based on the present expedition is worth mentioning. Suru valley is exceptionally the only place in the whole LehLadakh that is known to possess a good medicinal plant wealth plant like Ephedra gerardiana, Physochlaina prasalta, Picrorhiza kurroa, Artemesia martima, Podophyllum hexandrum, Carum carvi, Aconitum heterophyllum, Hyoscyamus niger yeild excellent plant drugs. Presently, Ephedra the source of Ephidrine hydrochloride, Ephidrine alkaloid, Physachlain and Hyoscyamus crude source of Atropine sulphate and Carcum the important only source of cumin used in Indian System of Medicine and flavouring agent for wines and soaps grown wildly in the valley in large quantity. Our country imports these active constituents in large quantity. These plant source used to be burned as fuel by inhabitants.

The active constituent can be extracted by planning the pilot plants in this area because transportation of crude drug in bulk would be a costly affair. After the extraction of active constituent at site the woody portion after drying can be utilised as fuel. The dried ripe fruits of corum can be collected in huge quantity and be cultivated in this region. Besides these, there are several medicinal plants like Orchis latifolia, Rheum emodi etc. growing in abundance which are in a great demand on large scale by the pharmaceutical industries. So by the planted collection and extraction of active constituent of medicine plant the foreign exchange which is being incurred for procurement of these drugs can be saved in lakhs. The soil and climate of these valleys are more suitable for the large scale propagation of Lupus humilus (Hop plant) used in liquor industry.

\section{Opportunities in developing the medicinal plants sector}

For developing the 'herbal industries', the northern India possesses a rich diversity of medicinal plant species across the various forest types along an altitudinal gradient (as discussed in the use and diversity of medicinal plants). Such a high diversity of medicinal plants would be helpful for further scientific research on exploring their medical efficacy, value addition, and use in curing various old and new diseases. India has already established a reputation as a low-cost manufacturer of high quality generic drugs in the global market. This fact can be used as an important tool for the marketing of herbal products produced in India. It is expected that India's aim to build a golden triangle between traditional medicine, modern medicine, and modern science will be a boon for developing the traditional herbal medicine and the medicinal plants sector.

\section{Existing policies}

In the National Five Year Plans of India, the medicinal plants sector has been identified as an integral part of the Indian System of Medicine and Homeopathy.

In 1983, the National Health Policy recognized that the large stock of health manpower in Ayurveda, Siddha, Unani, Homeopathy and Naturopathy had not been adequately utilized; therefore, steps need to be taken to move towards a meaningful integration of the indigenous and modern systems of medicine.

The Planning Commission and the National 
Medicinal Plants Board (NMPB) of the Government of India have prepared a policy document on the promotional and commercial aspects of the medicinal plants sector. In addition, the NMPB has prioritized 32 and Planning Commission has enlisted 24 medicinal plant species for research and development in order to meet the desired aim of the medicinal plant sector. The Biodiversity Act 2002 has framed many rules for sustainable utilization of medicinal plants and to mitigate the chances of bio-piracy.

\section{Institutional support}

In India, many government and nongovernment organizations have had the focused attention on improving the medicinal plants sector. Opportunities for funding have been created to assist the person who is willing to work and to build capacity of the medicinal plants sector. According to the mandate of NMPB, the projects may be submitted for funding within two major schemes: viz., a promotional scheme and a commercial scheme. The major thrust areas within the promotional scheme are:

1) Survey and inventory of medicinal plants

2) in-situ conservation and ex-situ cultivation of selected medicinal plants

3) Production of quality planting material

4) Diffusion of knowledge through education and communication

5) Promotion of global and domestic market system and

6) Strengthening research, development and manpower.

Conclusion of the study are as follows

With the advancement in science and technology the state of J\&K can make use of her rich biodiversity to sustain the livelihood of the poor farmer by sustainable production and utilization of medicinal and aromatic plants for the value added products.

The success of medicinal plants sector mainly depends on the awareness and interest of the farmers as well as its other stakeholders, supportive government policies, availability of assured markets, profitable price levels, and assess to simple and appropriate agro techniques.

Sustainable use of ecosystems which support millions of rural communities as well as major industry.

The successful establishments of medicinal plants sector may help in raising rural employment, boost commerce around the world, and contribute to the health of millions

Sustainable use of medicinal and aromatic plants involves the pertinent aspects of protection, preservation, maintenance, exploitation, conservation and sustainable utilization.

\section{References}

Agarwal, A. 2005. Current issues in quality control of natural products. Pharma Times 37(6): 9-11.

Bedi, Y.S. 2007. Plant Biotechnology Division. Current Science 92(10): 13901399.

Bhat, K.K.S. 1997. Medicinal and plant information databases. In: Medicinal Plants for Forests Conservation and Health Care. Non-Wood Forest Products, FAO, Rome, pp: 158.

Bodeker, G. 2003. Traditional medical knowledge, intellectual property rights and benefit sharing. Cardozo Journal of International and Comparative Law 11(2): 785-814

Chauhan, N.S. 1999. Medicinal and Aromatic Plants of Himachal Pradesh. Indus 
Publishing Company, New Delhi, pp: 21-25.

Govaerts, R. 2001. How many plants species are there? Taxon 50: 1085-1090.

Hoareau, L. and DaSilva, E.J. 1999. Medicinal plants: a re-emerging health aid. Electronic Journal of Biotechnology, Chile, 2: 56-70

Joshi, H.C., Arya, S.C. and Samant, S.S. 2001. Diversity, distribution and indigenous uses of plant species in Pindari area of Nanda Devi Biosphere Reserve. Indian Journal of Forestry 24(1): 514-536.

Kaul, M.K. 1997. Medicinal Plants of Kashmir and Ladakh. Temperate and Cold Arid Himalaya. Indus Publishing Company, New Delhi pp: 44-85.

Kaul, M.K. 2010. High altitude Botanical from Northwest Himalaya. Indian Journal of Traditional Knowledge 1: 18-25.

Khanuja, S.P.S., Kalra, A. and Singh, A.K. 2007. Survey of Indian agriculture: Need for a strategic approach. The Hindu pp: 186-190

Lange, D. 2002. The role of East and Southeast Europe in the medicinal and aromatic plants trade. Medicinal Plant Conservation. 8: 14-18

Madhuri, S. and Pandey, G. 2009. Some anticancer medicinal plants of foreign origin. Current Science 96(6): 779-783.

Pal, M. and Samant, S.S. 2005. Diversity, Distribution and Conservation of economically important medicinal and Aromatic plants of the Indian Himalayan region. G.B. Pant Institute of Himalayan Environment and Development, Kosi, Almora pp: 1-9

Paliwal, G.S. and Badoni, A.K. 1990. Ethnabotany of the Hill Tribes of Uttar Kashi: Medicinal Plants. J. Econ. Tax. Bot. 14(2): 421-442.

Parrotta, J. 2002. Conservation and sustainable use of medicinal plant resources -An international perspective. Paper presented at the World Ayurveda Congress, Kochi, Kerala, pp: 1-4.

Pushpangadan, P. 2006. Important Indian Medicinal Plants of Global Interest. Proceedings - International Conference on Botanicals, Kolkata, India, pp: 94109.

Pushpangadan, P. and George, V. 2008. Survey of Indian Agriculture: The opportunities are unlimited. The Hindu, pp: 186-190.

Roberson, F. 2008. Medicinal Plants at Risk. A Native Plant Conservation Campaign Report, pp: 1-15

Rodgers, W.A. and Panwar, H.S. 1998. Planning a wildlife protected area network in India. Wildlife Institute of India, Dehradun 1: 7-10.

Samant, S.S. and Dhar, U. 2002. Diversity, endemism and economic potential of wild edible plants of Indian Himalaya. Int. J. Sustain Dev. World Ecol. 4: 179191.

Samant, S.S., Dhar,U. and Palni, L.M.S. 2003. Medicinal plants of Indian Himalayas: Diversity Distribution Potential values. Gyanodya Prakashan, Nainital. pp:12-14.

Sharma, P.K. and Lal, B. 2005. Ethnobotanical notes on some medicinal and aromatic plants of Himachal Pradesh. Indian Journal of Traditional Knowledge 4(4): 424-428.

Schippmann, U., Leaman, D.J. and Cunningham, A.B. 2004. Impact of cultivation and Gathering of Medicinal Plants on Biodiversity: Global Trends and Issues. Rome: Inter-Department Working Group on Biology Diversity for Food and Agriculture, FAO.

Singh, M.P. and Somade. 2005. Indian Medicinal Plants. Satish Serial Publishing House, Azadpur, Delhi, pp: 1-5.

Tiwari, S. 2008. Plants: A rich source of 
Herbal Medicine. Journal of Natural Products 1: 18-25.

Upadhyaya, R.C. 2008. Agrotechniques of
Medicinal Plants. Anmol Publications Pvt. Ltd. New Delhi, pp: 79-91.

\section{How to cite this article:}

Sonam Dawa, Padma Gurmet, Tsewang Dolma, Tsering Angdus, Tashi Stobgais and Thinles Tharpa. 2018. Status of Medicinal and Aromatic Plants in the State of Jammu \& Kashmir. Int.J.Curr.Microbiol.App.Sci. 7(12): 2597-2615. doi: https://doi.org/10.20546/ijcmas.2018.712.295 